\title{
Design of low power triangle type stair cleaning machine
}

\section{XIN Haiyan ${ }^{1}$, LI Chengxiang $^{2}$, PENG Peng $^{1}$}

1. Southeast University Chengxian College,Nanjing,Jiangsu 210088,China,

2. North information control group Co, Ltd. Nanjing,Jiangsu 211153,China

Keywords: Low power consumption; triangle wheel; stair;Sweeping machine

\begin{abstract}
The traditional way of manual cleaning stairs, not environmental protection, cleaning efficiency and low, cleaning workers time and labor, in order to solve this problem, the design of a low power MSP430 series single chip staircase. The cleaning machine realizes the combination of the triangular roller and the ordinary wheel to realize the plane movement of the cleaning machine and the down stairs sweeping action. Multi sensors are used to collect the information of road surface, drive the stepper motor module, realize the function of location identification, automatic obstacle avoidance and so on. Through the steering gear to adjust the angle of the sweeping brush, so as to achieve the purpose of cleaning the stairs. The experimental results show that the cleaning machine can realize intelligent path identification, obstacle avoidance, downstairs and cleaning function, stable and reliable operation, with small structure, light weight, low cost, etc., has a certain practical value.
\end{abstract}

\section{Introduction}

With the development of society, more and more intelligent systems are beginning to take the place of human labor, and they are becoming more and more automated and intelligent [1]. The traditional way of manual cleaning is not only environmental protection, but also low cleaning efficiency. In such a special public environment, it is necessary to replace the traditional manual labor with high efficiency of cleaning tools, a large area of one-time cleaning and easy operation. Takahisa Kakudou et al. Proposed a kind of box climbing robot, which is a combination of climbing and cleaning, and uses the flip up and down way of the [2,3]. He Lianyun $[1,4]$ proposes the use of a parallelogram deformation characteristics achieve downstairs cleaning function of robot, its control core is STC89C51 SCM, response speed and information processing ability is insufficient. Therefore, this paper designed a low-power triangular wheeled stairs cleaning machine, the triangle roller and ordinary round combination, to achieve cleaner horizontal movement and downstairs, a brush cleaning mechanism for cleaning function of stairs on the ground. The cleaning machine with low power consumption MSP430 series processor, sensor detection with combination stair information, identify the location and obstacle avoidance functions, by controlling the stepper motor and steering action, so as to control the cleaning machine for walking, and a brush cleaning action.

\section{Design of the Overall scheme of the low power triangle stair sweeper}

\subsection{Working principle of the cleaning machine}

The working principle of the cleaning machine mainly includes: cleaning operation, garbage disposal, operation protection, etc.

-Cleaning operation mode: The sweeping machine adopts the combination of the triangle wheel and the common wheel, from top to bottom of the stairs slowly from one section to the next section of the operation steps, cleaning machine cleaning brush will run back together with the cleaning machine, and on the stairs under the action of gravity for cleaning. When the cleaning machine runs to the junction of the stairs and floor, identify the current location signal through the sensor, then control the stepper motor to make the cleaning machine turned 90 degrees, moving to the next section of stairs to work, always repeat the above process until the cleaning the stairs. The working track of the sweeper is shown in figure 1.

-Garbage disposal: The size of the cleaning machine is small, the garbage collection will 
increase the weight of the cleaning machine, is not conducive to reducing power consumption. Therefore, the use of garbage into the garbage disposal. When the machine is running, the garbage has been swept from the bottom to the bottom of the stairs, which can reduce the difficulty of the structure design and system energy consumption.

-running protection mode: The working environment of the cleaning machine is special, in order to reduce the harm to the cleaning machine, the cleaning machine adopts the design mode of meeting people and stopping. The use of infrared probe detection within a certain range of personnel activities, in order to achieve obstacle avoidance function.

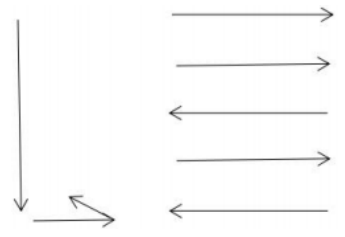

Fig.1 Working track of the sweeper

\subsection{Cleaning machine main technical parameters and working mode}

The main technical parameters and working modes of the sweeper are shown in table 1.

Table 1 the main technical parameters and working mode of the triangle wheel sweeper

\begin{tabular}{cc}
\hline item & Main technical parameters and working mode \\
\hline Running structure form & Wheel \\
Overall platform height & $190 \mathrm{~mm}$ \\
Overall platform width & $350 \mathrm{~mm}$ \\
Maximum travel speed & $0.3 \mathrm{~m} / \mathrm{s}$ \\
Turning radius & $500 \mathrm{~mm}$ \\
Cleaning method & Sweep while walking \\
Cleaning machine function & Automatic cleaning, intelligent obstacle avoidance \\
Working mode & and location identification \\
& Sweep up and down, Sweep before and after \\
\hline
\end{tabular}

\subsection{Design of General circuit of the Cleaning machine}

The hardware circuit includes the power supply, the sensor module, the stepper motor and its drive module, the steering gear drive module. The overall circuit diagram of the sweeper is shown in figure 2.MSP430F149 is selected as the cleaning machine core microprocessor, MSP430 series microprocessor of TI company is a special emphasis on the 16 bit ultra low power mixed signal processor, long time work and is suitable for the battery powered. The microprocessor with its low power consumption, strong processing ability, rich on-chip peripheral modules, convenient and efficient way of development and other characteristics, has been widely used in the portable instrument, intelligent sensor, practical testing instruments, such as motor control field ${ }^{[5]}$.

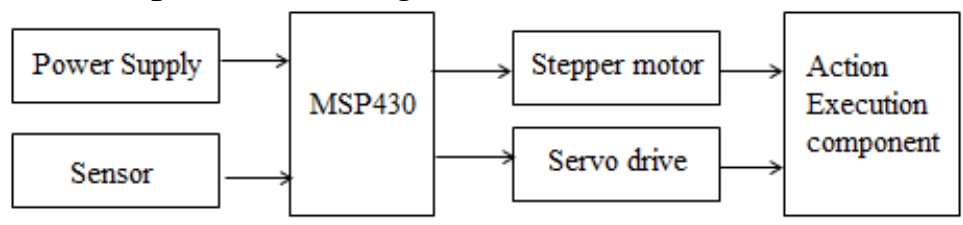

Fig.2 Overall circuit diagram of the Cleaning machine

\section{Design of the mechanical structure of the low power triangle wheel cleaning machine}

The mechanical structure of the cleaning machine mainly includes: climbing floor chassis, cleaning forearm, cleaning brush, etc.. In order to ensure that the sweeper can run smoothly on the stairs, the chassis adopts a combination of a triangular wheel and a common wheel. The circuit board is mainly used to install power supply, driver module, signal conversion module, as shown in figure 3. Cleaning machine is the main component of the floor of the triangle wheel, the use of the principle of three rounds of coaxial, cleaning machine to achieve the stable operation of the step road, as shown in figure 4. The installation of bearings with two inner diameter 8MM, not only can make the triangle wheel and the cleaning machine skeleton reasonable connection, and can reduce the resistance of the sweeper, reduce power consumption, as shown in figure 5 . In the system, the 
motor is more reasonable, so that the motor can make the cleaning machine has a simple and clear visual effect on the space structure, and the motor mounting bracket is shown in figure 6 . The brush is used to clean the rubbish on the stairs. It is made of acrylic board, as shown in figure7. Cleaning brush installation platform as shown in Figure 8, the component is mainly used to clean the brush connection, but also part of the system circuit installation platform. UG software design with the chassis effect diagram shown in figure 9 . The mechanical structure of the chassis is shown in figure 10.

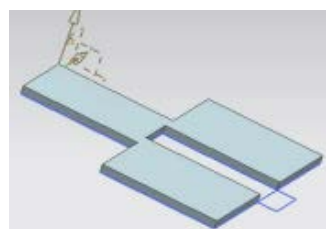

Fig. 3 Circuit board of cleaning machine

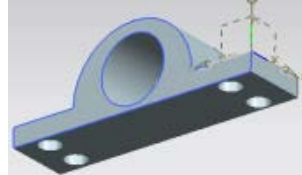

Fig.5Cleaner assembly bearing

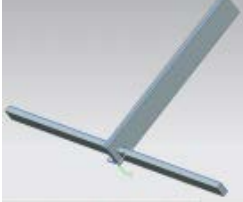

Fig.7Cleaning brush

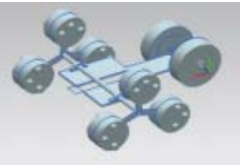

Fig. 9 UG renderings

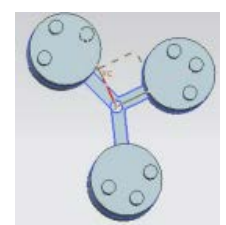

Fig. 4 Triangle wheel of cleaning machine

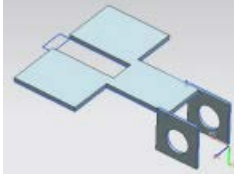

Fig.6 Cleaning machine motor mounting frame

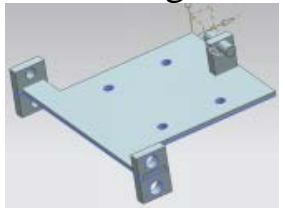

Fig.8 Cleaning brush installation platform

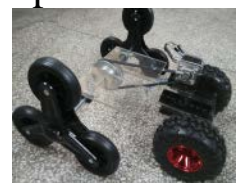

Fig. 10 Chassis physical map

\section{Design of the hardware circuit of the low power triangle wheel cleaning machine}

\section{1 sensor module}

The sensors used in this paper are mainly photoelectric switch and tilt sensor. The photoelectric switch is infrared sensor, mainly using the principle of light reflection, the switch head has a pair of infrared receiving tubes and tubes, when an object is within the sensing range of the probe, the launch tube emits light reflected by the object is received by the receiving tube, thereby changing the state of the circuit. The tilt sensor is the use of conductive copper ball in the trigger circuit, when the sensor is tilted in the conductive bead in the cylinder in its internal rolling, so as to realize the change of angle judgment. In order to solve the problem that the output level of the sensor can not be read directly by the processor, the level conversion module is added in the system to realize the handshake between the sensor and the processor. The sensor is connected with the processor, as shown in figure 11.

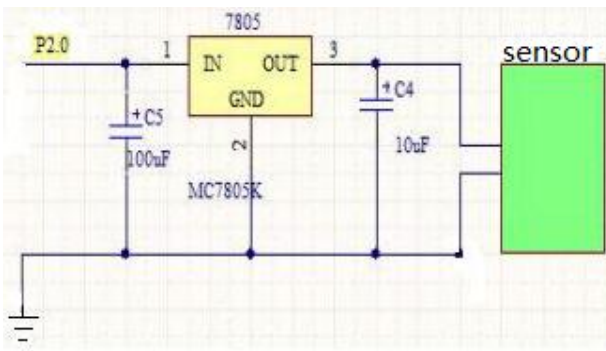

Fig.11 Sensor and MSP430F149 connection schematic

\subsection{Infrared sensor module}

In order to minimize the damage caused by the cleaning machine, the cleaning machine is equipped with an infrared sensor module. Infrared sensor module and MSP430F149 connection 
schematic, as shown in figure 12. When someone is in the probe sensing range, the probe inside the PNP three tube disconnection makes the circuit is disconnected, the microcontroller P1.0 pin is low, so that the stepper motor control signal failure. Make the machine stop running, to protect themselves.

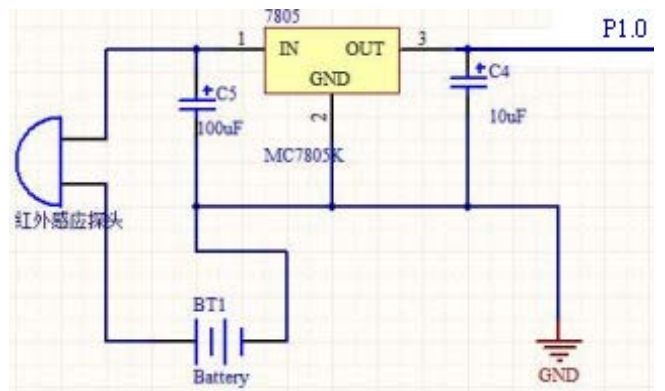

Fig. 12 Schematic of infrared sensor module and MSP430F149 connection

\subsection{Stepping motor and driving module}

Cleaning machine is used for beauty Beng high torque $12 \mathrm{~V}$ six sub line two stepper motor drive module, motor drive using L298N. Stepper motor drive schematic, as shown in figure 13.

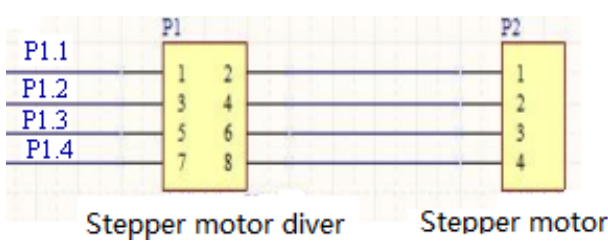

Fig. 13 Schematic diagram of stepper motor drive

\subsection{MG996R steering gear drive module}

The steering gear drive module is mainly used to control the steering gear, it uses the specific coding chip to change the resistance value of the circuit to realize the precise control of the steering angle. MG996R type steering gear is a kind of 180 degree rotation angle steering gear, which has the characteristics of accurate angle and low noise. In fact, as shown in figure 14. The steering gear control, there are many methods, such as using SCM IO port output PWM to control steering, can also use the servo drive plate is connected with the $10 \mathrm{~K}$ variable resistor, resistor can be adjusted by rotating the rotation angle of the steering gear. In this paper, PWM wave steering control, steering control circuit schematic diagram, as shown in figure 15.

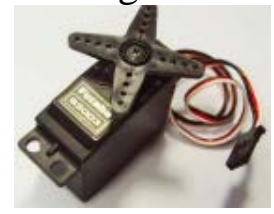

Fig. 14 MG996R type steering gear

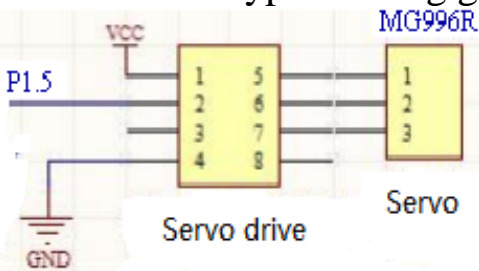

Fig. 15 Schematic diagram of steering gear control circuit

\section{Design of the software of the low power triangle wheel cleaning machine}

Cleaning procedures include: main program, interrupt program, stepper motor driver, steering gear control program.

\subsection{Design of the main program}

The main flow chart of the cleaning system is shown in figure 16. 


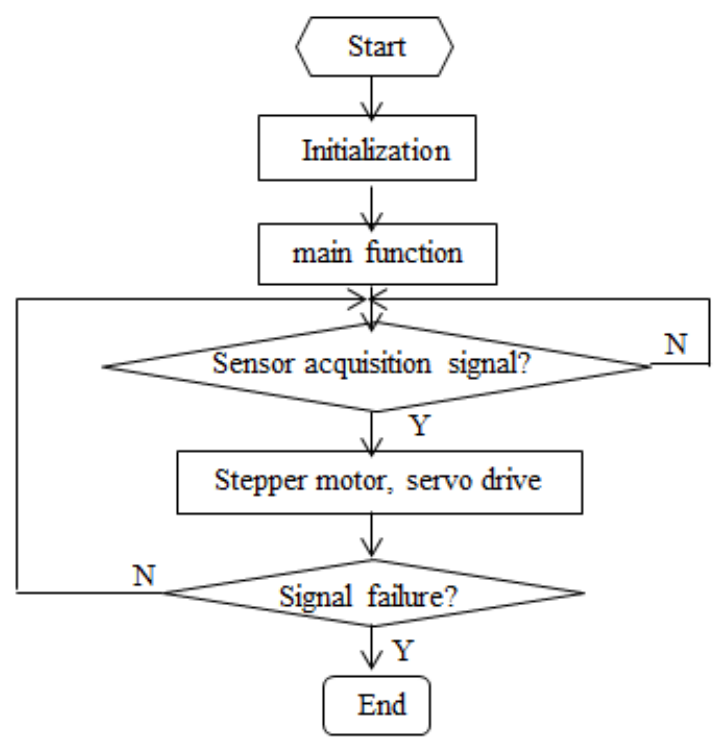

Fig. 16 The main program of the flow chart

\subsection{Design of Stepper motor driver}

The PWM control step motor is adopted in the cleaning machine control system. PWM wave control stepper motor timing diagram, as shown in figure 17. The speed control of stepping motor can be changed by changing the duty cycle of PWM wave. Cleaning machine for motor using single step control method, using IO simulation PWM wave of motor control. Motor control flow chart, as shown in figure 18.

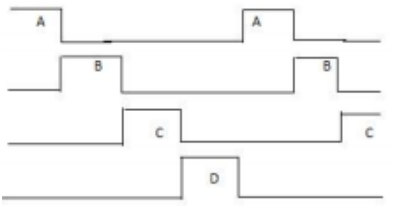

Fig. 17 The diagram of PWM wave control of stepper motor timing

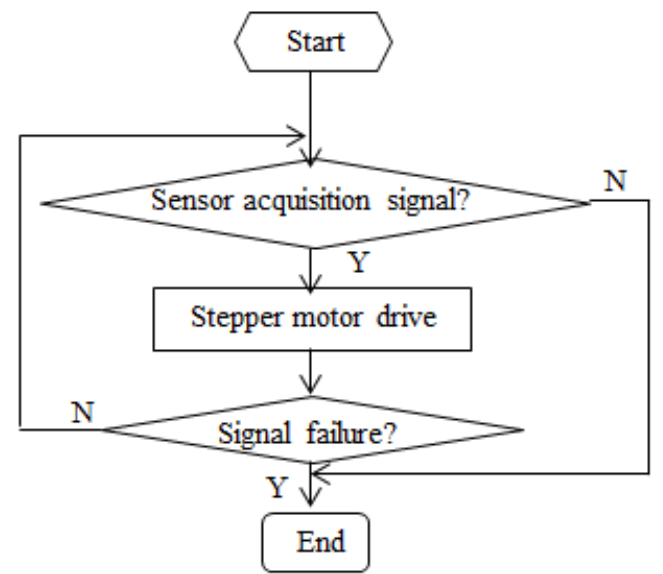

Fig. 18 Flow chart of stepping motor control

\subsection{Design of Servo control program}

MG996R servo control structure, as shown in figure 19.

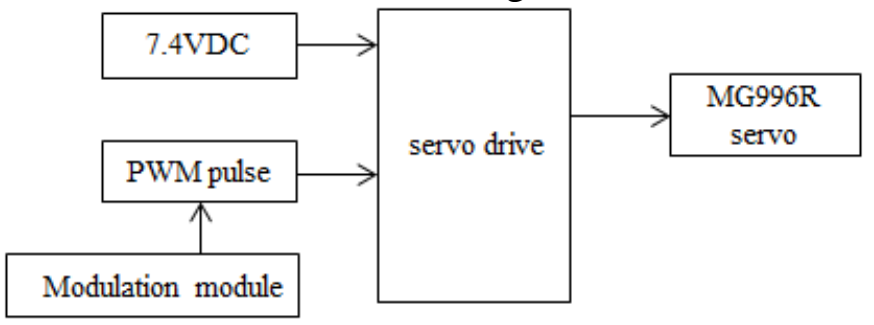

Fig. 19 The structure of steering gear control

Steering control flow chart, as shown in figure 20. 


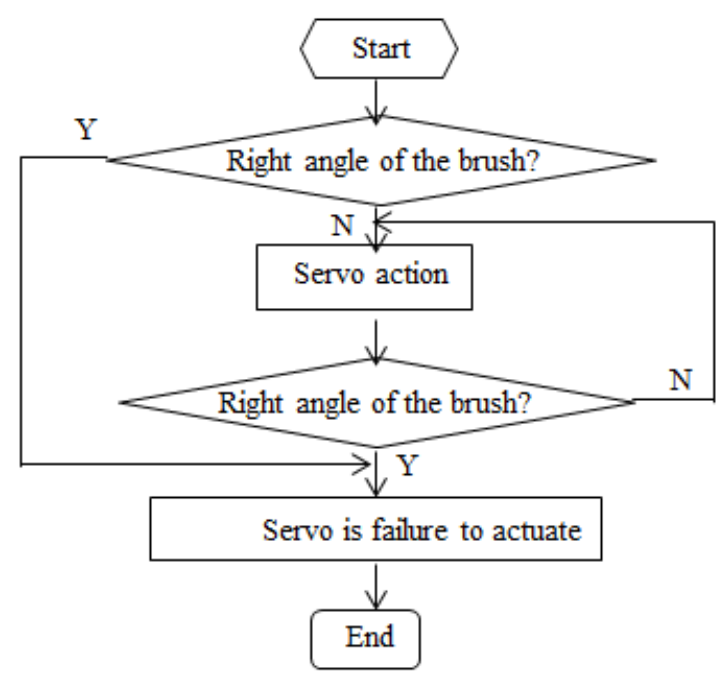

Fig. 20 Flow chart of steering gear control

\section{Conclusion}

Low power wheeled stair sweeper with delta MSP430 series single-chip microcomputer as control core, integrated photoelectric sensor module, stepper motor and servo module, and the corresponding software program under the control of the stairs collection information, the cleaning machine cleaning work and walk from top to bottom. The experimental results show that the cleaning function of different stair height, with the speed of $0.3 \mathrm{~m} / \mathrm{s}$ walking, and location identification, automatic obstacle avoidance function, low power consumption, small volume, light weight, stable and reliable operation, can reduce the staircase corridor cleaning labor intensity and improve labor efficiency, reduce the cost, provides a simple and convenient cleaning appliance for environmental protection workers, has a certain application value.

\section{References}

[1] Lianyun. Building cleaning robot design [J]. agricultural equipment and vehicle engineering, 2012,50 (8): 71-73.

[2]T. Kakudou, K. Watanabe and I. Nagai. Study on mobile mechanism for a stair cleaning robot design of translational locomotion mechanism[C].11th International Conference on Control, Automation and Systems,2011:1213-1216.

[3]T. Kakudou, K.Watanabe and I. Nagai. Mobile mechanism of a climbing robot for cleaning and locomotion on stairs[C].SICE Annual Conference,2012:145-148.

[4]He Lianyun. Model of biped robot walking and gait planning of [J]. information technology and information technology, 2008, (2): 64-66.

[5]XIN Haiyan.Research on the monitoring system of automobile tire pressure [D]. Changchun: Changchun University of Science and Technology, 2009.

[6]Yao Xingtian, Ma Yonglin, Zhang Lei, Zuo Yong. Structure and control system design of lifting telescopic corridor cleaning robot [J]. mechanical design and manufacture, April 2014. 